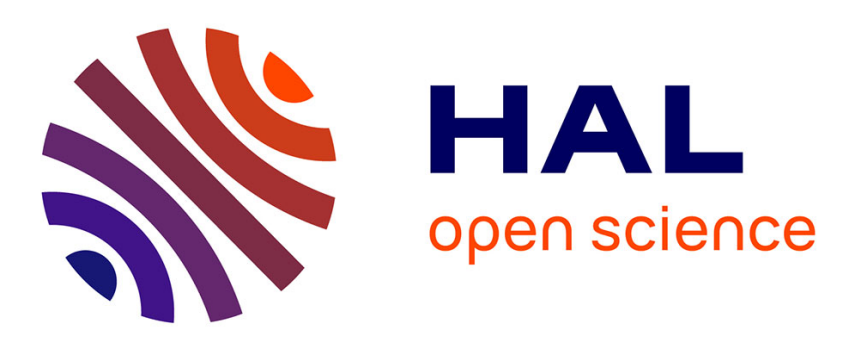

\title{
Des camps de squatters au nettoyage urbain: processus et enjeux de catégorisation autour du slum A Delhi
}

\author{
Véronique Dupont
}

\section{To cite this version:}

Véronique Dupont. Des camps de squatters au nettoyage urbain : processus et enjeux de catégorisation autour du slum A Delhi. Année Sociologique, 2018, 68 (1), pp.39-66. 10.3917/anso.181.0039 . ird01967655

\section{HAL Id: ird-01967655 \\ https://hal.ird.fr/ird-01967655}

Submitted on 1 Jan 2019

HAL is a multi-disciplinary open access archive for the deposit and dissemination of scientific research documents, whether they are published or not. The documents may come from teaching and research institutions in France or abroad, or from public or private research centers.
L'archive ouverte pluridisciplinaire HAL, est destinée au dépôt et à la diffusion de documents scientifiques de niveau recherche, publiés ou non, émanant des établissements d'enseignement et de recherche français ou étrangers, des laboratoires publics ou privés. 
Voir version éditeur publiée dans: L’Année Sociologique, Dossier thématique «L'expulsion: une nouvelle forme de gouvernement des sociétés urbaines ?» coordonné par Thomas Aguilera, Florence Bouillon, Martin Lamotte, 2018 (avril), no 68-1, pp. 39-66.

\title{
DES CAMPS DE SQUATTERS AU NETTOYAGE URBAIN : PROCESSUS ET ENJEUX DE CATEGORISATION AUTOUR DU SLUM A DELHI ${ }^{1}$
}

\author{
Véronique DUPONT
}

\section{Résumé}

A partir de recherches menées à Delhi, cet article propose une réflexion sur les désignations des quartiers d'habitat précaire illégaux - les slums - et les interventions-expulsions qui les visent. Les variations de perception et du vocabulaire autour de l'expulsion reflètent la position du locuteur (urbaniste, magistrat, activiste du droit au logement, habitant), ses registres de référence et la visée de ses discours. La thèse défendue est celle d'un effet de la catégorisation des slums et de leurs habitants sur le traitement qui en est fait. Nous montrons comment des catégorisations majoritairement disqualifiantes sont associées à des mises en accusation de situations irrégulières et de criminalisation, l'ensemble fondant des projets d'intervention. L'examen d'une opération de rénovation en cours dans un quartier précaire permet d'illustrer la manière dont les différents acteurs perçoivent et qualifient une même intervention et de souligner les divergences, y compris parmi les résidents. La partie conclusive replace les démolitions de slums dans le contexte plus large des transformations urbaines et des modes de gouvernance privilégiés.

Mots clés : Bidonvilles ; squatters ; démolition ; expulsion ; opération de rénovation ; désignation ; catégorisation ; gouvernance urbaine ; Delhi ; Inde.

\section{From squatter settlements to urban cleansing:} Processes and issues in the categorisations around the "slum" in Delhi Abstract

Based on research conducted in Delhi, this article offers insights and thoughts on the different designations of the illegal precarious settlements - the "slums"- and the interventions-evictions targeting them. The perceptions and the terminology around the evictions vary with the speakers and reflect their positions (whether urban planners, magistrates, housing rights' activists, inhabitants), their reference registers and what their discourses aim to accomplish. The thesis defended is that the categorisation of the slums and their inhabitants impacts the way they are treated. We show how categorisations that are predominantly disqualifying go hand in hand with indictments for irregular situations and with criminalisation, and are the basis for intervention projects. The examination of a rehabilitation project currently underway in a precarious settlement illustrates how the different actors perceive and qualify the same intervention, and highlights divergences, including among the residents. The concluding part places slum demolitions in the broader context of urban transformations and of the preferred modes of governance.

Key words: Slums ; squatters; demolition; eviction; rehabilitation; designation; categorisation ; urban governance; Delhi; India.

\footnotetext{
${ }^{1}$ Cet article se fonde sur des recherches initiées dans le cadre de deux projets collectifs : (i) le projet SETUP («Exclusion sociale, territoires et politiques urbaines. Une comparaison Inde-Brésil») financé par l'Agence nationale de la recherche de 2007 à 2010, et (ii) le projet Chance2Sustain (Urban Chances-City growth and the sustainability challenge) financé par la Commission européenne ( $7^{\text {ème }}$ Programme-cadre pour la recherche et le développement) de 2010 à 2014. J'ai également bénéficié de soutiens institutionnels, logistiques et financiers de l'IRD, du Centre de Sciences Humaines de New Delhi et du Centre for Policy Research de Delhi. Les enquêtes sur le site de Kathputli Colony à Delhi ont été conduites avec M.M. Shankare Gowda que je remercie pour son inestimable collaboration.

Je tiens également à remercier Thomas Aguilera, Florence Bouillon, Martin Lamotte, Pierre Lascourne ainsi que les experts anonymes pour leurs commentaires et suggestions précieuses.
} 


\section{Introduction}

Slums, jhuggi-jhopri clusters, camps de squatters, empiètements, ... ; slum clearance, nettoyage, épuration, éradication, expulsion, démolition, accaparement de terrain, évacuation, relocalisation, réinstallation, réhabilitation, opération de relogement: les désignations des quartiers d'habitat précaire illégaux des métropoles indiennes sont multiples; le sont tout autant les désignations des interventions publiques qui visent ces quartiers et impliquent une forme d'expulsion. Cet article propose une réflexion sur ces désignations et catégorisations et leurs effets sur les politiques.

L'étiquetage n'est pas anodin; il est consubstantiel à la construction du slum comme problème public ${ }^{2}$. Gilbert (2007) a dénoncé les dangers de l'utilisation du mot «slum», en raison de la confusion que ce terme entretient entre le problème physique lié à un habitat précaire, de mauvaise qualité, et les caractéristiques de ses habitants. Il montre comment la stigmatisation des habitants des slums encourage indirectement les démolitions et expulsions. Roy (2011), dans un article au titre évocateur de "Slumdog cities », souligne également la tendance à criminaliser les " informalités subalternes » et la violence considérable du pouvoir étatique qui en résulte (ibid., p. 223). Cet article se situe dans la lignée de ces analyses. La thèse défendue est celle d'un effet de la qualification des slums sur le traitement qui en est fait. Elle s'inscrit dans l'approche de la construction des problèmes publics selon le modèle du «naming, blaming, claiming» (Felstiner, Abel, Sarat, 1980-81). En appuyant notre argumentation sur des recherches menées à Delhi, nous montrerons comment des catégorisations majoritairement disqualifiantes sont associées à des mises en accusation de situations irrégulières et de criminalisation, l'ensemble fondant des projets d'actions expulsion, réinstallation ou rénovation de quartiers d'habitat précaire. Une lecture inversée du lien entre catégorisation des slums et politiques d'expulsion, que nous évoquerons, propose cependant de considérer la catégorisation comme le produit - et non la cause - de politiques urbaines contenant en germe l'expulsion.

En Inde, le terme de slum est utilisé de façon extensive dans les documents officiels, la presse et les travaux universitaires. Il recouvre au moins deux grandes catégories d'habitat. Selon la loi d'urbanisme de 1956, le Slum Areas (Improvement and Clearance) Act, les slums sont définis comme les secteurs urbains où les immeubles sont "impropres à l'habitation », souffrent de « l'étroitesse des rues », sont « délabrés, congestionnés, mal ventilés, mal éclairés ou dépourvus d'équipements sanitaires ${ }^{3}{ }^{3}$. Cette définition, qui s'inspire des textes britanniques sur les slums, visait d'abord les taudis de quartiers anciens et dégradés, et ne fait pas référence au statut d'occupation. La seconde catégorie de slums recouvre des regroupements d'habitations précaires, des auto-constructions en matériaux de récupération plus ou moins consolidées avec le temps, des huttes ou baraques appelées jhuggi-jhopries à Delhi. Cet habitat se caractérise par une insuffisance d'infrastructures et de services urbains de base et, en règle générale, par une occupation illégale des terrains. Dans cet article, nous nous intéresserons uniquement à cette seconde catégorie de slum, celle que l'on pourrait traduire par bidonvilles illégaux, désignés comme squatter settlements (camps de squatters), bastis ou jhuggi-jhopri bastis/clusters (regroupements de huttes).

Dans un premier temps, nous explorerons les correspondances entre, d'une part, la manière de désigner ces bidonvilles -et de qualifier leurs habitants- et, de l'autre, le type et la désignation des interventions-expulsions qui les ciblent. Nous montrerons comment les variations du vocabulaire et les perceptions autour de l'expulsion reflètent la position du

\footnotetext{
${ }^{2}$ Nous nous référons ici aux effets de qualification mis en évidence par Becker (1963) et à la théorie de l'étiquetage développée par Gusfield (1963), en reprenant les concepts utilisés en sociologie des problèmes publics (Neveu, 2015).

${ }^{3}$ The Slum Areas (Improvement and Clearance) Act, 1956, Chapter II.
} 
locuteur (urbaniste, magistrat, activiste du droit au logement, habitant), ses registres de référence, et la visée de ses discours. Nous analyserons également les variations terminologiques des modes d'intervention-expulsion en mettant à jour leur articulation avec l'évolution des perceptions, et avec la dimension que l'acteur-locuteur souhaite souligner. Dans un second temps, nous examinerons le cas d'une opération de rénovation en cours dans un quartier précaire de Delhi, Kathputli Colony. Cette échelle fine nous permettra d'illustrer plus concrètement la manière dont les différents acteurs perçoivent et qualifient une même intervention, et de souligner les divergences y compris entre différents groupes de résidents, en fonction des intérêts de chacun et des influences subies. Dans une partie conclusive, nous replacerons les démolitions de slums et l'étude de cas dans le contexte plus large des transformations urbaines à l'œuvre et des modes de gouvernance urbaine privilégiés. L'article analyse donc les variations lexicales : afin de mettre en évidence les correspondances entre la manière de désigner les bidonvilles et leurs habitants et les interventions qui les visent, nous reprendrons délibérément le vocabulaire utilisé par chaque acteur-locuteur. A contrario, le terme de bidonville sera utilisé ici comme catégorie générique de manière neutre, sans connotation particulière. De même pour le terme " expulsion », dont l'équivalent anglais n'est pas utilisé dans le contexte étudié.

Nos analyses s'appuient sur une combinaison de données secondaires et de données de première main. Les sources documentaires, mobilisées essentiellement pour la première partie de l'article, comprennent: des documents d'urbanisme et de politiques publiques (DDA, 1957, 2007; GNCTD, 2003-04), les mémoires d'un administrateur qui fût à la tête de l'agence de planification urbaine de Delhi (Jagmohan, 1978), le rapport d'une commission d'enquête sur les abus commis pendant l'état d'urgence de 1975-77 (Shah Commission of Enquiry, 1978), diverses statistiques produites par les administrations en charge des programmes à destination des bidonvilles (référencées plus précisément au cours du texte), des jugements emblématiques (High Court of Delhi, 2002, 2010; SCC, 2000), et des rapports d'organisations de défense du droit au logement (HLRN, 2009, 2011, 2013, 2014; IPTEHR, 2005) ${ }^{4}$. Les données primaires sont issues de nos propres enquêtes de terrain à Delhi. Une première enquête, conduite en 2008 et 2009, ciblait des familles délogées et exclues des programmes de réinstallation, avec des entretiens approfondis auprès de familles en provenance de 26 sites de bidonvilles démolis répartis sur le Territoire de la capitale ${ }^{5}$. Une seconde série d'investigations, menée depuis 2009, porte sur le projet de rénovation de Kathputli Colony. Elle comprend une soixantaine d'entretiens approfondis (auxquels s'ajoutent de nombreuses discussions informelles) avec divers types d'acteurs (résidents, leaders locaux, responsables d'organisations non gouvernementales [ONG], activistes, ingénieurs et urbanistes de l'agence de planification urbaine, dirigeants au sein de la firme immobilière, consultant privé, politiciens, associations de résidents des quartiers voisins), des observations directes de terrain, y compris de réunions et manifestations, complétées par une revue de presse et de sites internet.

\section{De l'étiquetage des quartiers précaires à leur démolition}

L'histoire de la planification urbaine à Delhi depuis l'indépendance (1947) apporte une première démonstration des effets de désignation des bidonvilles, que nous illustrerons à partir de quelques périodes clés : la mise en place du premier plan d'urbanisme à la fin des années 1950, les abus de l'Etat d'urgence (1975-77), les politiques mises en œuvre dans les années 1990 et 2000. Précisons que le schéma directeur d'urbanisme pour Delhi est voté par

\footnotetext{
${ }^{4}$ Les traductions en français de citations originales en anglais sont les nôtres.

${ }^{5}$ Pour plus de détails sur la méthodologie de cette enquête, voir Dupont (2009).
} 
le Parlement de l'Union et a force de loi. Il est élaboré et mis en œuvre par la Delhi Development Authority (DDA), l'agence de planification urbaine placée sous la tutelle du Ministère du développement urbain au niveau du gouvernement fédéral. La DDA représente directement les pouvoirs publics et constitue la première autorité urbaine à Delhi ${ }^{6}$.

Dans cette première partie, nous examinerons, outre le langage des urbanistes, celui des magistrats, à partir de quelques affaires emblématiques, ainsi que le registre mobilisé par les militants du droit au logement pour dénoncer les évictions forcées. Le programme de rénovation de bidonvilles initié à Delhi dans la dernière décennie (2007-2017) sera examiné en seconde partie à partir d'une étude cas, afin de poursuivre l'analyse des variations lexicales à un niveau plus fin.

\section{Les représentations des urbanistes}

Dans les premiers documents d'urbanisme de la DDA, les bidonvilles illégaux, désignés sous le terme hindi de bastis, sont constitués en objet de stigmatisation. Ils sont décrits comme « des tâches pestilentielles dans le paysage urbain, des concentrations insalubres, de délinquance et de vices, [...] à la fois une disgrâce et une source de danger pour toute la ville » (DDA, 1957, p. 223). Leurs habitants sont perçus comme des migrants « ignorant des modes de vie urbain et persistant dans leurs anciens modes villageois [qui] ont rendu la condition des bidonvilles encore plus sordide » (DDA, 1957, p. 223).

De telles perceptions vont longtemps perdurer, et la criminalisation des habitants se systématiser. Alors que les termes hindis de basti et jhuggi-jhopri basti (regroupements de huttes) soulignent la dimension précaire de la structure d'habitation, l'introduction d'une dimension délictueuse se traduit par un nouveau vocabulaire. Les habitants sont qualifiés par les urbanistes de "squatters" (des occupants illégaux) et d'encroachers (ceux qui empiètent abusivement sur le territoire des autres), et leur habitat de squatters settlements (camps de squatters). La criminalisation de ces «informalités subalternes » sera exacerbée dans les années 2000 par les discours des tribunaux (voir infra).

Ces perceptions sont associées dès les années 1950 à un objectif d' "élimination des slums et des camps de squatters » annoncé dans les documents préparatoires du Plan d'urbanisme de Delhi (DDA, 1957, p. xx). L'amélioration des conditions de vie dans les camps de squatters, notamment par la fourniture de services de base était également envisagée dans ce premier plan (1962-1981) pour des raisons d'hygiène et de santé publique. Elle était cependant conçue comme une mesure temporaire en attendant que les squatters soient « relocalisés » sur des trames d'accueil (des parcelles assainies non construites).

Perceptions et désignations disqualifiantes ont des effets destructeurs lorsqu'elles s'intègrent dans des politiques étatiques de nettoyage et d'embellissement de la ville à grande échelle. L'Etat d'urgence, mis en place de juin 1975 à janvier 1977 et qui entraîna une parenthèse dans le régime démocratique de l'Inde indépendante en offre une démonstration dramatique (Shah Commission of Enquiry, 1978). Nettoyer et embellir la capitale, « faire de Delhi une ville belle et moderne » a d'abord signifié des démolitions massives - l'ordre du jour étant de «nettoyer la ville rapidement ${ }^{7}$. L'état d'urgence avait établi les conditions exceptionnelles d'un pouvoir absolu de démolition de structures jugées « empiètements non conformes " ou encroachments. L'ampleur et le rythme des démolitions ont été

\footnotetext{
${ }^{6}$ Cette situation particulière tient au statut de Delhi : un Territoire de l'Union qui, bien que pourvu d'une assemblée élue et d'un gouvernement depuis 1991, n'a pas acquis toutes les prérogatives d'un Etat de la fédération. Le gouvernement central maintient le contrôle sur le foncier et les politiques urbaines par l'intermédiaire de la DDA.

${ }^{7}$ Ces citations sont extraites de propos tenus par B.R. Tamta, le commissaire municipal de l'époque (Shah Commission of Enquiry, 1978, p. 79).
} 
spectaculaires. En moins de deux années, plus de 700000 personnes ont été délogées de force des slums de la vieille ville et des bastis et envoyées dans des lotissements dits de réinstallation dans les périphéries - de fait, de simples trames d'accueil. Les autorités urbaines de l'époque, la DDA et notamment son vice-président Jagmohan, n'eurent aucune considération pour la violence des déplacements forcés. Ainsi Jagmohan se félicitait-il de cet « accomplissement » et du succès d'une telle politique montrant la voie à suivre (Jagmohan, 1978, p. 54). De fait, des politiques similaires d'éradication des slums se sont répétées jusqu'aux années 2000 (voir infra).

Le cadrage effectué par Jagmohan, un protagoniste actif des démolitions, et son administration n'est pas étranger à ce zèle de purification urbaine. Jagmohan considérait les « squatters » comme une menace pour la ville entière - pour son esthétique, sa santé publique, sa vie sociale et sa productivité économique (ibid., 1978, pp. 57, 63). Leurs lieux d'habitation sont traités de «bidonvilles puants » (stinking slums - ibid, p. 31), de " taches affreuses » (ugly spots -ibid., p. 36) dont il fallait débarrasser le paysage urbain au profit de l'aménagement de parcs (ibid: 38). On retrouve l'obsession sanitaire, récurrente chez les urbanistes depuis la période coloniale (Dupont, Ramanathan, 2007), associée ici à une priorité donnée aux espaces verts sur l'habitat des pauvres - un conflit qui ressurgira dans les années 1990 et 2000.

\section{Novlangue et visée du discours - les expulsions oblitérées}

L'intitulé des différents programmes à destination des jhuggi-jhopri clusters mis en œuvre par les autorités urbaines à Delhi depuis les années 1960 jusqu'aux années 2000 révèle une évolution dans le vocabulaire désignant le même type d'intervention. L'approche privilégiée pendant toute cette période fut en effet la démolition des camps de squatters et la relocalisation de leurs habitants sur des sites viabilisés (resettlement colonies). Il ne s'agissait pas de programmes de relogement mais de simples réinstallations sur des parcelles assainies sans aucune aide à la reconstruction. Le premier programme lancé en 1960 s'intitulait le Jhuggi-Jhopri Removal Scheme, indiquant une élimination des habitations précaires. Depuis les années 1990, on parle de programme de «relocalisation» des jhuggi-jhopries: sans véritable changement de politique, ce nouveau vocable met l'accent sur la dernière phase de l'intervention, la réinstallation sur des sites alternatifs. Le nombre de familles relocalisées est alors présenté dans les rapports du département des Slum[s] \& Jhuggi-Jhopri[es] de la Municipalité de Delhi comme une performance louable (voir par exemple GNCTD, 2003-04). Un tel cadrage tend à oblitérer la première phase de l'intervention, celle souvent brutale de la démolition et de l'éviction avec un recours systématiques aux forces de police. Or, pour les habitants, la relocalisation signifie tout d'abord la destruction de leur maison et une expulsion forcée de leur lieu et milieu de vie. Par ailleurs, l'imposition de critères restrictifs d'éligibilité aux programmes de relocalisation (l'application d'une date butoir d'installation sur le site initial et le versement de charges ${ }^{8}$ ) génère un nombre très important d'exclus. Les conditions de mise en œuvre du programme - négligences, dysfonctionnements et pratiques de détournement - entraînent en outre l'exclusion de familles pourtant éligibles (Dupont, 2010). En conséquence, derrière les chiffres officiels de familles relocalisées, on doit également évaluer le nombre, considérablement plus élevé de familles expulsées, pour beaucoup d'entre elles mises à la rue sans aucune solution alternative?

\footnotetext{
${ }^{8}$ Les sommes à verser couvrent un dépôt de sécurité, les dépenses de relocalisation et le droit d'occupation pour dix ans, représentant largement plus du double du revenu mensuel de la plupart de ces familles.

${ }^{9}$ L'analyse du devenir des familles exclues des programmes de réinstallation a fait l'objet d'un autre article (Dupont, 2010).
} 
Quelques chiffres permettent de préciser l'ampleur des démolitions et du processus d'exclusion dans la capitale indienne. En 1998, trois millions de personnes habitaient dans 1100 jhuggi-jhopri clusters de taille très variable, occupant essentiellement des terrains publics et représentant $27 \%$ de la population de la ville de Delhi dans ses limites administratives ${ }^{10}$. Selon les statistiques officielles, de 1990 à 2008, 221 jhuggi-jhopri clusters ont été démolis et 65000 ménages ont été « relocalisés »" ${ }^{11}$, soit environ 325000 personnes, avec un dernier pic de démolition pendant les réaménagements urbains en préparation aux Jeux du Commonwealth de 2010. On estime cependant autour d'un million le nombre de personnes déplacées de 1998 à 2010 par ces démolitions (Ghertner, 2010). In fine, plus de la moitié des ménages délogés ont été exclus des programmes de réinstallation (Bhan, Shivanand, 2013). Autre indicateur révélateur de l'ampleur et des conséquences de ces exclusions, la population des sans abri à Delhi a augmenté de $68 \%$ entre 2000 et 2008, atteignant au minimum 150000 personnes restant à la rue, ou prenant refuge la nuit dans les asiles municipaux ${ }^{12}$.

Dans le cadre du programme modifié de réinstallation pour les habitants de jhuggijhopries, approuvé en 2010 par le gouvernement de Delhi, l'attribution d'appartements remplacera l'attribution de parcelles. A nouveau, la liste des conditions d'éligibilité (notamment la preuve de résidence avant une date butoir et la prise en compte des seuls occupants du rez-de-chaussée des habitations) conduisait à l'exclusion d'une majorité des familles. Le Delhi Urban Shelter Improvement Board (DUSIB) ${ }^{13}$ a estimé que, dans la liste des 16 groupements de jhuggi-jhopries identifiés en 2011 pour une réinstallation prioritaire, seulement $40 \%$ des familles seraient éligibles pour l'attribution d'appartements, laissant la grande majorité des habitants délogés sans alternative ${ }^{14}$. Ce constat, ainsi que la pression exercée par des organisations militantes, ont amené le gouvernement à assouplir les conditions d'éligibilité en 2013, puis à nouveau en 2015 après l'investiture dans l'Etat de Delhi du parti populaire Aam Admi (parti de l'homme ordinaire). La sélectivité des programmes et les processus d'exclusion demeurent cependant consubstantiels aux politiques mises en place à Delhi, les expulsés exclus s'avérant les victimes des interventions publiques.

\section{Le poids des mots dans les cours de justice}

Dans un contexte d'intervention croissante du judiciaire dans la gouvernance des villes indiennes depuis les années 1990, notamment dans les politiques environnementales et vis-àvis des slums, le langage des cours de Justice - et ses implications - mérite une attention particulière. Le discours des tribunaux envers les slums s'est durci dans les années 2000. Certains jugements prononcés à Delhi ont contribué à délégitimer et criminaliser les habitants des slums, sans les reconnaître comme victimes de l'incapacité des pouvoirs publics (la DDA) à remplir leur obligation d'assurer un logement aux catégories défavorisées, conformément aux stipulations du Plan d'urbanisme de Delhi. Deux affaires emblématiques à cet égard sont présentées ci-dessous ${ }^{15}$. Nous les avons retenues pour leur portée : les décisions de justice prononcées concernent en effet toute l'agglomération de Delhi et l'ensemble de ses slums.

\footnotetext{
${ }^{10}$ Source : Slum and Jhuggi-Jhopri Department, Municipal Corporation of Delhi - le département en charge de la mise en œuvre des programmes à destination des bidonvilles. Voir Dupont (2008) pour une analyse critique des données sur la population des camps de squatters à Delhi et leur démolition.

${ }^{11}$ Source : Slum and Jhuggi-Jhopri Department, Municipal Corporation of Delhi.

${ }^{12}$ Ces estimations sont basées sur les enquêtes d'ONG travaillant auprès des sans abri (IGSSS, 2012).

${ }^{13}$ Ce Board fut créé en 2010 sous la tutelle du gouvernement du Territoire de Delhi pour reprendre les fonctions de l'ex-Département des Slums et Jhuggi-Jhopri de la Municipalité.

${ }^{14}$ Source: " Minutes of the fifth Board Meeting of the DUSIB, 19 December 2011 », Site internet du DUSIB: http://delhishelterboard.in (téléchargé le 31 décembre 2016).

${ }^{15}$ Pour une analyse détaillée de ces affaires, voir Dupont et Ramanathan (2008).
} 
La première cause, Almitra Patel vs Union of India, est un litige d'intérêt public porté à la Cour Suprême par un environnementaliste contre les pouvoirs publics. Elle traitait initialement du problème de l'enlèvement des déchets solides dans Delhi, et aboutit à un ordre de la Cour aux autorités urbaines d'épurer la ville non seulement de ses ordures mais également de ses slums (SCC, 2000). L'argumentaire est fondé sur le lien supputé entre les slums et la génération de déchets solides, construit comme un problème à traiter en priorité :

«La création de slums [...] doit être empêchée. [...] Ce sont les ordures et les déchets solides générés par ces slums qui requièrent un traitement des plus expéditifs et sur une base prioritaire » (ibid., p. 685).

Ce raisonnement n'est fondé sur aucune preuve empirique, et va à l'encontre des résultats d'études sur la production d'ordures ménagères selon le quartier et le niveau de revenu (Srishti, 2002). Un second argument repose sur l'assimilation du squatter à un voleur afin de délégitimer les programmes de réinstallation :

«Récompenser un squatter (encroacher) sur un terrain public par un site alternatif gratuit, c'est comme donner une récompense à un pickpocket » (SCC, 2000, p. 685).

Outre la métaphore préjudiciable, cet argument oublie que l'accès à une parcelle dans un lotissement de réinstallation n'est jamais gratuit pour la famille déplacée ${ }^{16}$. Ce jugement révèle ainsi un biais de raisonnement, dans la mesure où il n'est fondé ni sur des faits avérés, ni sur un raisonnement juridique, mais sur un jugement de valeur disqualifiant. In fine, la cour ordonne que des mesures appropriées soient prises pour améliorer les conditions sanitaires dans les slums existants, jusqu'à ce qu'ils soient rasés et les terrains récupérés (ibid., p. 688).

Le projet d'éradication des slums est réitéré dans l'affaire Okhla Factory Owners' Association [une association d'industriels] vs Government of National Capital Territory of Delhi (High Court of Delhi, 2002). Dans cette cause, qui traite de la relocalisation des habitants de slums occupant des terrains publics, la Haute Cour de Justice de Delhi examine la légalité et la validité de la politique de réinstallation sur des trames d'accueil mise en œuvre par le Gouvernement de Delhi. Dans leur argumentaire, les magistrats accusent les habitants des slums d'avoir contrecarré le développement planifié de Delhi (ibid., para. 2). Alors que l'expropriation des petits fermiers à travers la procédure d'acquisition de terrains avait été justifiée au nom de l'intérêt public pour la planification urbaine, selon la Cour l'utilisation de tels terrains pour relocaliser « les personnes qui ont squatté et se sont introduits illégalement sur les terrains publics [...] équivaudrait à donner une prime à une telle malhonnêteté et aux empiétements de terrains » (ibid., para. 35). En conséquence, les magistrats invalident la politique alors en vigueur : " Aucun site alternatif ne devra être pourvu dans le futur pour l'éviction des personnes qui squattent sur des terrains publics » (ibid, para. 49) ${ }^{17}$. La description dans le jugement des habitants des slums comme des encroachers ${ }^{18}$, malhonnêtes et usurpateurs, des fardeaux pour le contribuable qui devrait supporter le coût des nouveaux terrains à acquérir pour leur réinstallation, peut être interprétée comme une stratégie discursive pour délégitimer les habitants des slums et dénier leur droit à un habitat en ville. C'est aussi un cadrage qui occulte l'obligation des pouvoirs publics de répondre aux besoins en logement des populations pauvres. En d'autres termes, c'est un encouragement à des expulsions sans aucune alternative ni compensation.

D'une manière générale, dans les années 2000, les slums ont été représentés dans les cours de justice comme sources de problèmes, et le «nouveau discours de nuisance » utilisé comme le principal outil argumentatif pour procéder aux démolitions de slums à Delhi

\footnotetext{
${ }^{16}$ Chaque famille éligible doit verser une contribution financière conséquente pour accéder à une parcelle (voir note 8).

${ }^{17}$ La brutalité de ce jugement conduisit à un appel en Cour Suprême et à un ordre suspensif.

${ }^{18}$ Le terme «encroacher », ou ses dérivés « encroachement» et «(to) encroach», figurent 66 fois dans les 18 pages du jugement.
} 
(Ghertner, 2015, p. 111). Dans de nombreux cas, les tribunaux sont intervenus en réponse à des pétitionnaires représentant les intérêts d'industriels ou d'associations de résidents de classe moyenne et supérieure, qui ont mis en avant des considérations environnementales et d'assainissement dans le cadre de litiges d'intérêt public pour demander l'évacuation des slums voisins.

A contrario, certains jugements plus récents basés sur des droits humains se sont appuyés sur une conception des habitants des slums comme citoyens à part entière, protégés par la Constitution indienne, pour affirmer le droit à un abri de ces derniers. C'est le cas de l'affaire Sudama Singh \& others vs Government of Delhi (High Court of Delhi, 2010), où les requérants étaient les habitants d'un bidonville démoli. Un tribunal de la Haute Cour de justice de Delhi rejeta l'argument du gouvernement de Delhi et de la Municipalité selon lequel les habitants de jhuggies ne méritaient pas d'être relocalisés sur des sites alternatifs lorsque leurs jhuggies occupaient les voies publiques, violant ainsi le droit de passage. Le tribunal opposa l'argument suivant : «Les habitants des jhuggies ne doivent pas être traités comme des citoyens de seconde zone» (ibid, para. 57). "Ce sont les citoyens qui aident le reste de la ville à vivre une vie décente, ils méritent la protection et le respect des droits à la vie et à la dignité que la Constitution leur garantit (ibid., para. 61) ».

Ces différents exemples montrent comment la perception des habitants des slums par les magistrats témoigne soit d'une adhésion aux préjugés caractéristiques de certaines époques, et aux valeurs et intérêts des classes dominantes, soit de leur dépassement dans une vision humaniste. Cette défense d'intérêts catégoriels ou au contraire leur rejet se traduit par la mobilisation d'un répertoire lexical au sein des décisions de justice dont les conséquences sur le sort de ces populations sont tout à fait concrètes - leur expulsion, ou leur protection ${ }^{19}$.

\section{Les militants du droit au logement et la dénonciation des évictions forcées}

Les organisations de défense du droit au logement mobilisent un vocabulaire spécifique, différent de celui des urbanistes et des autorités urbaines, en prenant comme cadre de référence les conventions internationales et les droits humains. L'Inde a ratifié presque toutes les conventions internationales relatives aux droits humains, dont le Pacte international relatif aux droits économiques, sociaux et culturels, qui détaille la question du droit à un logement décent. Si ces conventions sont loin d'être respectées (Kothari, 2003), elles fournissent néanmoins des instruments de droit international, un cadre de légal sur lequel ces organisations peuvent s'appuyer (HLRN, 2009).

Le concept convoqué au sujet des démolitions d'habitations précaires est celui d'expulsion -ou éviction- forcée, définie par l'ONU comme :

« l'éviction permanente ou temporaire, contre leur volonté et sans qu'une protection juridique ou autre appropriée ait été assurée, de personnes, de familles ou de communautés de leurs foyers ou des terres qu'elles occupent ${ }^{20} \gg$.

Une telle pratique est reconnue par le Haut Commissariat aux droits de l'homme de l'ONU comme constituant une violation flagrante des droits humains, notamment du droit à un logement convenable (ONU-Habitat, 2014, p. 1).

Les organisations telles que le Housing and Land Rights Network (HLRN) reprennent cet argument pour dénoncer les expulsions forcées, dont nous avons souligné l'ampleur à Delhi. Selon les cas, elles décrient l'absence de notification préalable aux habitants, les

\footnotetext{
${ }^{19}$ Des mécanismes similaires ont été mis en évidence dans les procédures d'expulsion des squats en France (Bouillon, 2010).

${ }^{20}$ Comité des droit économiques, sociaux et culturels, Observation générale $n^{\circ} 7$ (1997) relative au droit à un logement convenable: expulsions forcées. Citée dans : ONU-Habitat, 2014, p. 3.
} 
violences de la police et le recours à la force physique pendant les opérations de démolition, la perte des biens personnels, la perte des moyens de subsistance consécutive à la démolition de l'habitat, l'interruption de scolarité des enfants, l'absence de toute réinstallation proposée (notamment lors de certaines des démolitions qui ont précédé les Jeux du Commonwealth de 2010), ou les conditions inadéquates de la réinstallation. Les titres des rapports sont suffisamment éloquents : Swept off the Map. Surviving Eviction and Resettlement in Delhi (Menon-Sen, Bhan, 2008), Planned Dispossession: Forced Evictions and the 2010 Commonwealth Games (HLRN, 2011), Forced to the fringes. Disasters of 'Resettlement' in India (HLRN, 2014), ou encore, à propos de la démolition à Mumbai de 90000 habitations en quelques mois (de novembre 2004 à mars 2005) : Bulldozing Rights (IPTEHR, 2005). Un autre rapport, dénonçant des expulsions forcées et démolitions d'habitat à Bangalore, s'intitule Governance by Denial (HLRN-PUCL, 2013), pour caractériser un modèle de gouvernance urbaine qui nie le droit à la ville des pauvres, une critique qui pourrait s'appliquer à de nombreux autres cas en Inde. Nos propres enquêtes à Delhi auprès de familles délogées corroborent les effets d'appauvrissement cumulatifs consécutifs aux démolitions, spécialement dans le cas des familles exclues des programmes de réinstallation (Dupont, 2010).

\section{Une première conclusion : variations du répertoire et persistance des disqualifications}

Les variations terminologiques relevées autour des slums et de leur expulsion sont liées à la position des locuteurs, à leur manière d'appréhender les droits d'occupation des habitants des slums, et au projet d'action qu'il s'agit de justifier - ou de combattre. Cette première partie a également mis en évidence des usages modulés du vocabulaire associé à ces expulsions pour une même catégorie d'acteurs-locuteurs, en correspondance avec des évolutions normatives ou la visée de leurs discours à un moment donné.

Au-delà des variations lexicales, cet examen des discours tenus au cours des soixantedix années qui suivirent l'Indépendance de l'Inde met en évidence la persistance de perceptions négatives à l'encontre des slums et de leurs habitants. Les référents, normes et représentations des urbanistes, pour partie legs de la période coloniale ${ }^{21}$, se sont combinés aux préjugés de caste et de classe, en particulier vis-à-vis des basses castes et ex-intouchables, et plus généralement des migrants pauvres (Deshingkar, Farrington, 2009, p. 23), présents en grands nombres dans les bidonvilles. Les témoignages recueillis lors de nos enquêtes attestent de situations où transparaît le parti pris des agents municipaux et de la police contre les habitants des jhuggies, considérés comme des squatters, des citadins non légitimes, des résidents illégaux. "Ce sont comme des insectes, il faut les écraser sinon ils ne quitteront jamais cet endroit » : tels sont les propos attribués à un sous-commissaire de police au sujet de familles délogées en 2006 de leur habitat sur les berges de la rivière Yamuna, exclues des programmes de réinstallation et restées, faute d'alternative, sur le site du bidonville démoli. Ces propos nous ont été rapportés par un des habitants expulsés, témoin de cette conversation $^{22}$. "Nous sommes traités comme des animaux» se plaignent des habitants exclus des programmes qui ont essayé de faire valoir - en vain - leurs droits auprès de l'administration ${ }^{23}$. Ces témoignages, exemples parmi d'autres, confirment l'existence d'une stigmatisation génératrice de mépris et de violence, un processus également analysé par d'autres auteurs (Baviskar, 2003 ; Menon-Sen, Bhan, 2008).

Précisons cependant que la récurrence du terme de «squatters " n'est pas nécessairement associée à un déni de reconnaissance du droit à la ville de ces populations.

\footnotetext{
${ }^{21}$ Ce point n'a pu être approfondi dans le cadre limité de cet article. Voir Dupont et Ramanathan (2007).

${ }^{22}$ Interview dans le lotissement de réinstallation de Bhawana, Delhi, le 5 mars 2008.

${ }^{23}$ Interview dans un campement sur les berges de la Yamuna, Delhi, le 29 février 2008.
} 
Dans les écrits universitaires, l'utilisation de ce terme peut résulter d'un choix délibéré, pour souligner les conséquences de cette qualification. Mettre l'accent sur l'absence de droit légal de tenure, à l'instar de Datta (2012) dans son ouvrage The Illegal City, vise à rappeler la violence des catégorisations juridiques qui impacte la vie quotidienne des squatters. Il s'agit de la menace constante d'une expulsion forcée, mais aussi des difficultés permanentes pour avoir accès à l'eau ou pour assurer des fonctions corporelles vitales dans un habitat dépourvu d'équipement sanitaire.

\section{Un projet de rénovation « pilote » à Delhi : Kathputli Colony}

Cette deuxième partie se concentrera sur un cas de rénovation de quartier précaire à Delhi, le projet de Kathputli Colony ${ }^{24}$. Ce projet est emblématique car le premier de ce type un partenariat public-privé- à être mis en œuvre dans cette ville, inaugurant le nouveau modèle destiné à être répliqué. C'est également un projet majeur de par la taille de la population affectée, soit près de 20000 personnes. Nous dégagerons à nouveau les variations de perception et de terminologie selon la position du locuteur. En outre, nous analyserons comment les appréciations varient parmi les habitants eux-mêmes en fonction des intérêts particuliers de chacun, et de l'influence d'acteurs extérieurs au bidonville.

\section{La vision de l'agence publique de planification urbaine, la DDA}

Le Schéma directeur d'urbanisme pour Delhi à l'horizon 2021, promulgué en 2007, envisage une nouvelle stratégie pour répondre au défi des camps de squatters occupant des terrains publics (la quasi totalité d'entre eux à Delhi), désignés indifféremment sous le label de « squatter settlements 》, " squatter slums » ou "JJ (pour jhuggi-jhopri) clusters 》 (DDA, 2007, section 4.2.3). Reconnaissant les limites des programmes de relocalisation des squatters sur des trames d'accueil en périphérie (qui nécessitent des acquisitions considérables de terrains et éloignent les familles de leurs ressources économiques et sociales), la stratégie alternative est basée sur la fourniture de logements dans des immeubles à plusieurs étages, dans le cadre d'un partenariat public-privé en utilisant le foncier comme ressource. Concrètement, seule une partie du terrain occupé par le camp de squatters (au minimum $60 \%$ ) est utilisée pour la construction d'immeubles destinés à reloger ses habitants. Le reste du terrain dégagé revient au promoteur privé pour des constructions résidentielles ou commerciales mises en vente sur le marché à des fins de profit, une partie du produit de la vente permettant de financer les logements sociaux. Les attentes des autorités urbaines sont triples : «nettoyer» la ville de ses slums; fournir des logements à leurs habitants; et libérer la valeur des terrains occupés par les slums pour des usages plus lucratifs ${ }^{25}$. En 2008, la DDA identifia une première liste de $21 \mathrm{JJ}$ clusters en vue de leur rénovation selon le nouveau modèle, en projetant la construction de 37000 logements ; Kathputli Colony était le premier d'entre eux.

Pour la DDA, Kathputli Colony était un camp de squatters qui occupait un terrain de 5,22 hectares lui appartenant. Situé dans une zone centrale de Delhi, bien relié au reste de la ville par les transports en commun, le camp abritait fin 2013 environ 3500 ménages, dont un groupe important d'artistes populaires et d'artisans originaires du Rajasthan implantés à la fin

\footnotetext{
${ }^{24}$ Pour une analyse détaillée du contexte et des premières étapes de la mise en place du projet de rénovation, voir Dupont et al. (2014).

${ }^{25}$ Interview à la DDA avec un responsable du projet de Kathputli Colony (4 septembre 2015).
} 
des années $1960^{26}$. Depuis, d'autres groupes de migrants, en provenance de diverses régions, s'y étaient installés. Kathputli Colony était devenue un bidonville hétérogène où les conditions de logement très variables reflétaient les disparités socio-économiques entre habitants.

Le projet de relogement a été dévoilé aux habitants en février 2009 lors d'une cérémonie inaugurale en présence du ministre du Développement urbain : y fut annoncé un ensemble d'immeubles de 14 étages, comprenant 2800 appartements de deux pièces, d'une surface unitaire de $25 \mathrm{~m}^{2}$. La DDA présente ce projet de rénovation de bidonville comme un projet pilote de « réhabilitation sur site (in-situ rehabilitation) », inspiré du modèle appliqué à Mumbai depuis les années 1990, mais pionnier dans le contexte de Delhi. Pour une série de raisons qui ne pourront pas toutes être détaillées ici, la mise en œuvre du projet s'est avérée beaucoup plus longue et difficile que prévue. Elle a d'abord nécessité la construction d'un camp de transit, à charge du promoteur, situé à trois-quatre kilomètres du site de Kathputli Colony, pour accueillir les familles dans des unités d'habitation préfabriquées pendant la construction des immeubles de relogement. Le camp fut achevé début 2013, mais les premiers transferts de familles n'ont commencé qu'un an plus tard - la mise en œuvre du déplacement restant de la responsabilité de la puissance publique. La majorité des familles a résisté pendant près de quatre ans au transfert dans le camp de transit, jusqu'à l'expulsion forcée fin octobre 2017 des 2700 ménages restant en trois jours, sous le couvert d'environ 350 policiers. Les protestations des habitants devant l'entrée en action des bulldozers furent réprimées par des bastonnades et des grenades lacrymogènes. La démolition complète du bidonville annonce le démarrage prochain du chantier de construction.

Pour expliquer l'opposition au projet d'une partie des habitants, les chefs du projet à la DDA mettent en avant les intérêts particuliers de certains groupes, invoquant les "slum lords » qui louent de nombreuses jhuggies, les trafiquants de drogue et d'alcool, ceux qui viennent se réfugier après avoir commis crimes ou délits, ceux ou celles qui se livrent à la prostitution. Kathputli Colony est ainsi décrit comme «a den for drugs, a safe heaven for criminals and a place for prostitution $»^{27}$. Si des pratiques rentières et des trafics illicites y étaient effectivement repérables, la colonie ne pouvait se réduire à une zone de marginalité et de délinquance. On retrouve chez ces urbanistes un processus de disqualification classique par la mise en exergue de certains faits et figures repoussoir pour produire un récit englobant.

\section{La vision du promoteur privé}

Le promoteur privé qui a remporté le contrat pour le projet de relogement de Kathputli Colony l'annonçait en 2010 en ces termes dans sa lettre d'information à visée publicitaire : « un projet pilote pour Delhi dont l'objectif est de fournir de meilleures conditions de vie aux populations urbaines pauvres », [...] « une référence pour de nombreux projets avec le but ultime de faire de Delhi un Etat sans bidonville $»^{28}$. Plus récemment, à l'occasion d'une visite du camp de transit, le directeur général de la firme immobilière réitéra publiquement son engagement d'améliorer les conditions de vie des habitants : "Je vais construire des appartements tels qu'ils seront enviés par tous; tout le monde y compris le gouvernement considèrera ce projet de logements comme un modèle $»^{29}$.

\footnotetext{
${ }^{26}$ Kathputli en hindi signifie marionnette, Kathputli Colony est ainsi nommée pour ses nombreux marionnettistes.

${ }^{27}$ Interviews à la DDA avec un responsable du projet de Kathputli Colony (3 septembre 2014) et avec son successeur (4 septembre 2015).

${ }^{28}$ Source : "Raheja bags Delhi first slum redevelopment project", Impression- Quarterly Newsletter, Vol iv, Issue 1, New Delhi, January 2010 [https:/www.yumpu.com/en/document/view/25651059/impressions-rahejadevelopers, Site consulté en juin 2017].

${ }^{29}$ Déclaration faite le 14 avril 2017 par Raheja.
} 
Le promoteur utilisera $60 \%$ du terrain occupé par le bidonville pour construire et livrer gratuitement à la DDA le complexe de logements sociaux pourvu des aménités obligatoires. Pour rendre cet investissement rentable, le promoteur a un plan ambitieux. Sur les $40 \%$ du terrain lui revenant, il projette de construire une tour résidentielle de luxe de 54 étages qui serait l'immeuble le plus haut de Delhi, ainsi qu'un complexe commercial, en joint venture avec une société de construction basée à Dubaï, celle qui a construit la plus haute tour du monde. Ainsi, derrière l'objectif social affiché, se profile une opération immobilière lucrative, que ne manqueront pas de dénoncer les opposants au projet.

\section{Le point de vue de habitants : des opinions diverses et divergentes}

Depuis son initiation, le projet de relogement a suscité parmi les habitants des réactions et opinions contrastées. Alors que certains l'ont considéré comme une occasion d'accéder à de meilleures conditions d'habitat, d'autres sont restés très sceptiques, ou franchement opposés au projet. Entre ces groupes aux opinions antithétiques, beaucoup se sont simplement résignés au cours du temps à accepter le projet, ne voyant pas d'autres issues. Pour tous, les sujets de préoccupation sont restés nombreux dans les huit années suivant l'annonce du projet. Le manque d'informations précises de la part de la DDA sur les modalités de sa mise en œuvre a suscité inquiétude et méfiance. Les critères d'éligibilité pour accéder à un appartement dans le complexe de relogement et les conditions financières, notamment la contribution des familles bénéficiaires, n'ont été clarifiés que très tardivement. En outre, la liste des ménages bénéficiaires a été divulguée seulement en février 2014, soit plus de trois ans après l'enquête d'identification de la DDA. Elle a révélé de nombreuses omissions (produit de négligence ou dysfonctionnement ?) qui ont renforcé les craintes d'exclusion du programme de relogement et donc d'expulsion définitive du lieu de vie - des craintes compréhensibles si l'on se réfère aux programmes précédents de réinstallation des squatters à Delhi.

Dans ce contexte, un groupe de leaders locaux a engagé des négociations actives avec la DDA en concentrant les demandes sur deux points : l'inclusion dans le programme des ménages éligibles qui avaient été omis ; et l'obtention de garanties formelles de relogement par un accord écrit avant de déménager dans le camp de transit. L'objet ici n'est pas de relater les différentes phases et modalités de mobilisation des habitants, ni la constitution de deux groupes antagonistes aux frontières fluctuantes, ceux qui s'opposent au projet et ceux qui le soutiennent. Nous chercherons plutôt à montrer les différents points de vue en présence, les variations de perception, qui sont fonction des craintes ou intérêts de chacun, ainsi que des influences subies.

\section{L'adhésion au projet : la promesse d'un avenir meilleur}

Les habitants favorables au projet de rénovation le considèrent comme une bonne opportunité pour améliorer leurs conditions de vie et sécuriser leur droit d'occupation. En d'autres termes, ils ont réalisé que le « droit de rester » dans un environnement très dégradé et insalubre n'était «pas un grand privilège» (Dewan Verma, 2002, p. 67). Les femmes en particulier souffrent du manque d'équipement sanitaire et doivent faire face aux difficultés quotidiennes d'approvisionnement en eau pour la maisonnée. Accéder à un appartement équipé d'eau potable au robinet, de toilettes, d'une salle de bain et d'une cuisine séparée tout ce qui fait défaut dans les habitations précaires d'un bidonville - est une promesse de confort et de modernité : "Au moins nos enfants apprendront à vivre dans un environnement moderne $»^{30}$. Résider dans un appartement est aussi considéré comme un gage de

\footnotetext{
${ }^{30}$ Interview d'un résident après son déménagement récent dans le camp de transit (30 mars 2017).
} 
respectabilité, pour échapper au stigmate du bidonville. Le projet de rénovation est ainsi perçu comme porteur de progrès et d'ascension sociale.

Sans surprise, l'adhésion au projet de relogement et les attentes vis-à-vis de celui-ci sont liées aux conditions initiales d'habitat. Ainsi les premiers groupes à avoir déménagé dans le camp de transit sont ceux qui vivaient dans les conditions les plus précaires, dans des baraquements exigus, en matériaux de fortune. Pour eux, une unité d'habitation de $12,5 \mathrm{~m}^{2}$ dans les préfabriqués du camp, avec sanitaires communs, représente déjà une amélioration par rapport à leur condition antérieure. Inversement, pour les familles les plus prospères, aux maisons consolidées qui furent condamnées à la démolition, le passage en camp de transit requiert l'acceptation d'une perte conséquente d'espace et de confort, voire de statut, et un ajustement nécessaire pendant une période transitoire, dans la perspective d'un avenir meilleur. L'adhésion finale au projet dépend aussi de la capacité de négociation de la famille avec les pouvoirs publics et le promoteur. Plusieurs leaders ont ainsi réussi à obtenir dans le camp de transit une unité d'habitation (et donc un appartement dans le futur complexe) pour chaque membre adulte de leur famille élargie. Les opposants au projet dénoncent aussi les sommes versées par le promoteur pour convaincre certains leaders dont l'adhésion est recherchée en raison de leur influence sur les membres de leur communauté. Nos enquêtes de terrain apportent un certain crédit à ces allégations.

Enfin, pour certains habitants qui ne dissimulent pas leurs intentions, le projet de relogement est perçu comme une très bonne occasion de spéculation immobilière et d'enrichissement personnel ${ }^{31}$. Un appartement situé dans une zone centrale de Delhi est un bien immobilier qu'il sera possible de revendre rapidement avec profit - en détournant les interdictions de revente prévues par la DDA pendant les dix premières années.

\section{Les opposants au projet : un habitat inadapté, une procédure inique}

Les opposants au projet mettent en avant deux types principaux d'arguments : le projet est inadapté à leurs besoins, et c'est un accaparement de terrain scandaleux, qui s'effectue à leurs dépends.

La résistance la plus forte au projet de rénovation est provenue du groupe dominant des artistes et artisans. Ils considèrent que vivre en appartement dans des tours d'une quinzaine d'étages n'est pas adapté à leur mode de vie et à leurs activités professionnelles. Cette inquiétude est partagée par les familles qui possèdent des volailles ou des chèvres, composante intégrale de leur économie domestique, ou encore des singes dressés pour des spectacles de rue. Beaucoup auraient préféré l'attribution d'un terrain, leur permettant de construire graduellement et d'ajouter des étages en fonction de l'agrandissement de leur famille, ou pour louer une pièce comme source de revenu. De facto, les résidents n'ont pas été invités à participer à la conception du projet de relogement, ils ont seulement été informés a posteriori. Aucun effort, tant de la part de l'agence publique que du promoteur, n'a été fait pour prendre en considération leurs points de vue et leurs besoins. Ce cas corrobore les travers habituels des projets de rénovation ou de réinstallation des bidonvilles : un manque de participation effective des habitants, et une conception ignorant la dimension économique des bidonvilles comme lieu d'activités essentielles aux moyens d'existence de leurs habitants.

Le second facteur d'opposition au projet s'est exprimé de manière beaucoup plus véhémente. Les opposants dénoncent un accaparement de terrain (land grab) au détriment des habitants dépossédés de « leur» terre, et un scandale immobilier au profit du promoteur. Les habitants de Kathputli Colony ne se considéraient pas comme des squatters, mais comme des occupants légitimes, de longue date, d'un terrain qu'ils avaient contribué à aménager, et

\footnotetext{
${ }^{31}$ Interview d'un leader local, un des premiers à avoir déménagé dans le camp de transit (5 avril 2017).
} 
intrinsèquement lié à leur condition d'existence. «Zameen Hamari Jaan Hai / Cette terre est notre vie » était ainsi le titre d'une pièce montée par les marionnettistes de la colonie avec le soutien d'artistes extérieurs, pour mettre en scène leur menace d'expulsion et sensibiliser le public à leur cause, juste quelques mois après la première entreprise d'évacuation du bidonville par la DDA (en février 2014).

« Pourquoi devrions-nous payer la DDA pour un appartement? C'est notre terre » est un argument fréquemment entendu dans le groupe des opposants. Certaines ONG et des militants venus soutenir la lutte des opposants ont fourni de nouveaux arguments de défense basés sur les droits fondamentaux des citoyens. Ces activistes incitèrent les habitants à revendiquer leur droit à la terre et au logement, à travers des communiqués de presse et des slogans scandés lors des rassemblements de protestation. Par exemple:

«Arrêtez les accaparements de terre! Garantissez les droits à la terre pour tous les habitants de Kathputli Colony! ${ }^{32}{ } \gg$

« La terre est notre droit ».

Ce dernier slogan était récurrent lors des protestations organisées quotidiennement dans la colonie pendant plusieurs mois en 2017, entre la deuxième tentative de la DDA pour transférer les habitants dans le camp de transit et la démolition finale du bidonville. Alors que l'évacuation fut annoncée cinq jours en avance, les ONG et mouvements politiques qui prônaient la résistance n'ont pourtant pas mobilisé leurs militants pour venir soutenir les habitants face aux forces de l'ordre et aux bulldozers.

Des leaders de Kathputli Colony et les activistes, mieux informés des conditions du contrat conclu entre l'agence publique et le promoteur privé, dénoncent des illégalités et un favoritisme au bénéfice du promoteur. Ces accusations ne sont pas dénuées de fondement; elles se réfèrent à un audit interne de la DDA qui relève des irrégularités dans l'exécution du projet, des avantages indus en faveur du promoteur (notamment un prix d'acquisition nettement inférieur au prix du marché), et une part de terrain excédentaire attribué au promoteur $^{33}$. Par ailleurs, des rumeurs prétendent que les conditions mêmes du processus d'appel d'offres avaient été truquées pour favoriser la firme qui a remporté le contrat (Raman, 2015). En résumé, les opposants au projet de rénovation qualifient celui-ci d'escroquerie foncière et immobilière.

Les points de vue opposés examinés ci-dessus dans le contexte d'une même intervention urbaine ne doivent pas être considérés comme figés et isolés les uns des autres. Nos observations de terrain, suivies sur plusieurs années, ont mis en évidence des glissements et recompositions d'alliance entre les partisans du projet et ses adversaires parmi les résidents. Mis à part un noyau dur d'irréductibles dans chaque camp, les positions d'une majorité d'habitants ne sont pas statiques, ni réductibles à leurs situations résidentielles. Elles ont au contraire évolué en fonction d'influences extérieures, notamment celles d'activistes, ou de politiciens, mais aussi des stratégies de l'agence publique d'urbanisme et du promoteur. Ces stratégies ont combiné des phases de négociation et persuasion, le versement de sommes d'argent pour convaincre les leaders locaux influents, et des opérations d'intimidation (forte présence policière lors des entreprises d'évacuation, arrestations des opposants, bulldozers positionnés aux abords du bidonville) jusqu'à la démolition finale.

\footnotetext{
32 "Stop land grab! Ensure land rights for all the residents of Kathputli Colony», Press Release, Hazard Centre "in consultation with the residents of Kathputli Colony", 7 February 2014.

${ }^{33}$ Audit Officer, "Irregularities in execution/development of Kathputli Colony", Audit Report, DDA Cell, Delhi Development Authority, March 2011.
} 


\section{Expulsions : moyen de développement des métropoles}

Dans cette partie conclusive, nous prendrons de la distance par rapport à l'étude de cas de Kathputli Colony pour replacer les démolitions de bidonvilles et les projets de rénovation dans le contexte plus large des stratégies de développement des métropoles indiennes et de leur mode de gouvernance.

Depuis une quinzaine d'années, l'ambition de développer les métropoles indiennes en villes de rang mondial a entraîné des restructurations majeures de leur espace, notamment des démolitions de bidonvilles de grande envergure. Le cas de Delhi est exemplaire à cet égard. La capitale indienne avait déjà une histoire de "nettoyage urbain » pour faciliter son développement. Nous avons évoqué les démolitions massives pendant l'Etat d'urgence de 1975-77. Les Jeux Asiatiques de 1982 ont également été précédés d'opérations d'embellissement et d'éradication des bidonvilles. L'organisation d'un nouvel événement sportif international, les Jeux du Commonwealth de 2010, a joué un rôle d'accélérateur dans les opérations de renouveau et d'embellissement urbain, au détriment des habitants des bidonvilles (Dupont, 2011). Deux ressorts sont à souligner dans ces processus d'expulsion.

Le premier est une logique d' " embellissement». Le bidonville ne trouve pas sa place dans le modèle d'une ville moderne, qui plus est aux aspirations de ville mondiale. Dans son ouvrage Rule by Aesthetic Ghertner (2015) a théorisé ce mode de gouvernance par un principe d'esthétique selon lequel la légitimité d'un territoire urbain repose sur des normes d'esthétique: les slums sont déclarés illégaux parce qu'ils paraissent illégaux, et sont considérés comme une nuisance visuelle à éliminer. Selon un tel cadrage, les démolitions des slums contribuent à l'amélioration de l'environnement. Si Ghertner situe sa thèse dans la première décennie du $\mathrm{XXI}^{\mathrm{e}}$ siècle, nous avons montré dans cet article la pertinence d'une approche par les perceptions et catégorisations pour les décennies antérieures également. L'appréhension négative des slums et la disqualification des squatters depuis les premiers plans d'urbanisme des années 1950 jusqu'aux jugements des tribunaux des années 2000 ont eu des effets destructeurs à Delhi.

La seconde logique d'expulsion également à l'œuvre dans la capitale indienne renvoie à la thèse d'accumulation par dépossession proposée par David Harvey (2008), appliquée au cas indien par des géographes radicaux à l'instar de Banerjee-Guha (2010); elle rejoint l'argumentation développée par Saskia Sassen (2016) dans son ouvrage Expulsion. Les forces du marché poussent à la récupération de terrains occupés par les bidonvilles, afin d'en tirer un meilleur profit. Cette stratégie de déverrouillage du foncier et de captation des plus-values foncières, est préconisée en Inde par les politiques du logement, notamment dans le cadre de partenariats entre secteur public et privé. Ainsi les programmes de rénovation de slums mis en œuvre par la DDA peuvent être décryptés comme un transfert des terrains publics squattés vers le secteur privé, favorisant la promotion immobilière haut de gamme (Raman, 2015, $\mathrm{p}$. 390). Appliquée à Mumbai depuis les années 1990, une telle politique a été critiquée pour avoir favorisé les intérêts du lobby immobilier et permis à une mafia foncière de prospérer, avec un risque de harcèlement des habitants des bidonvilles situés sur des terrains potentiellement lucratifs (Nijman, 2008 ; Weinstein, 2008).

Si l'expulsion des bidonvilles à Delhi peut s'analyser comme un mode de gouvernance urbaine fondé sur une double logique, certains auteurs interprètent la catégorisation même des habitats en illégal ou légal comme une technique pour gouverner (Bhan, 2013, p. 68), ou - en d'autres termes - un mode de gouvernance de l'espace urbain (Roy, 2005). Dans cette logique, la persistance des bidonvilles dans l'espace urbain n'est plus considérée comme un échec de la planification urbaine et de la mise en œuvre des politiques du logement, mais comme le produit du plan et des règlements d'urbanisme qui déterminent les catégorisations 
et en conséquence quels habitats auront droit de cité et lesquels sont voués à l'éradication (Bhan, 2013). Les expulsions sont alors un produit direct de la planification urbaine.

Cette planification au bulldozer a remodelé la structuration socio-spatiale de Delhi. Les démolitions de bidonvilles se sont accompagnées d'une relégation de leurs habitants en périphérie et ont favorisé un modèle de ségrégation socio-spatiale excluant les pauvres des espaces centraux et des « beaux quartiers » (Dupont, 2008). Cette dynamique a contribué à un changement d'échelle de la ségrégation: d'une ségrégation résidentielle à l'intérieur du quartier, lorsque les bidonvilles occupaient tous les interstices de l'espace urbain et étaient dispersés sur l'ensemble du territoire, vers une ségrégation socio-spatiale à l'échelle de l'agglomération urbaine ${ }^{34}$. En revanche, les nouveaux projets de rénovation sur site en partenariat public-privé, où l'habitat social pour les habitants de bidonvilles va jouxter des immeubles de grand standing, inaugurent du retour d'une ségrégation résidentielle à une échelle micro-locale.

Notre exploration des perceptions et qualifications autour des slums et des expulsions à Delhi suggère plusieurs enseignements en guise de conclusion. Elle invite tout d'abord à porter une attention particulière aux glissements entre catégorisation de l'habitat et caractérisation de ses habitants, ainsi qu'aux correspondances entre leurs qualifications et le traitement qui leur est réservé, les premières préfigurant souvent le second et servant de légitimation et de justification aux expulsions. La relation n'est cependant pas univoque : les stratégies de catégorisation des slums sont à la fois à l'origine de politiques d'expulsion, et un produit intrinsèque de la planification urbaine.

Chaque acteur de la scène urbaine a ses propres lunettes -ou œillères- pour voir une même réalité, et son propre registre lexical pour l'exprimer, qui traduit son idéologie, sa position, la visée de son discours et ses intérêts particuliers. Nous retrouvons une même variété de visions et d'expressions au niveau micro-local, sur la scène d'un projet de rénovation de bidonville, avec des divergences marquées y compris parmi les habitants selon leur situation résidentielle et les jeux d'autres acteurs intervenant. L'observateur est frappé par la persistance et la diffusion de figures disqualifiantes à l'encontre des habitants des bidonvilles : persistance dans le temps et diffusion parmi des catégories d'acteurs en position de force - urbanistes, magistrats, policiers. Et les défenseurs des droits humains de dénoncer au fil des décennies les mêmes préjudices pour les habitants expulsés.

In fine, l'analyste retiendra l'imbrication de plusieurs schémas explicatifs pour rendre compte de l'ampleur des expulsions de bidonvilles dans la capitale indienne. Les deux principales logiques identifiées - logique d'embellissement, et stratégie d'accumulation par dépossession - s'inscrivent dans des modes de gouvernance de l'espace et renvoient à une vision exclusive du développement urbain. Au-delà, les expulsions apparaissent participer d'une politique urbaine qui s'appuie sur les stratégies capitalistes des promoteurs et appuie ces dernières. Les investigations menées à Delhi, ainsi que l'expérience plus ancienne de Mumbai quant à l'implication des promoteurs privés dans les programmes d'éradication des bidonvilles, corroborent cette analyse.

Véronique DUPONT

Institut de Recherche pour le Développement (IRD), Centre d'études en sciences sociales sur les mondes africains, américains et asiatiques (UMR CESSMA, Université Paris Diderot - INALCO - IRD) veronique.dupont@ird.fr

\footnotetext{
${ }^{34}$ L'appréciation de la ségrégation socio-spatiale est tributaire de l'échelle à laquelle l'on se place. Voir Dupont (2004) pour une analyse détaillée du cas de Delhi sur cette question.
} 


\section{RÉFÉRENCES BIBLIOGRAPHIQUES}

Bhanerjee-Guha S. (ed), 2010, Accumulation by Dispossession. Transforming Cities in the New Global Order, Delhi, Sage.

Baviskar A., 2003, "Between violence and desire: space, power, and identity in the making of metropolitan Delhi”, International Social Science Journal, 55, 175, pp. 89-98.

Becker H., 1963, Outsiders, New York, The Free Press of Glence.

Bhan G., 2013, "Planned Illegalities. Housing and the 'Failure' of Planning in Delhi: 19472010”, Economic \& Political Weekly, 48, 24, pp. 58-70.

Bhan G., Shivanand S., 2013, “(Un)Settling the City. Analysing Displacement in Delhi from 1990 to 2007”, Economic and Political Weekly, 48,13, pp. 54-61.

Bouillon, F, 2010, «Le squatter, le policier, le juge et le préfet: procédures en actes et classement ad hoc », Déviance et Société, 34, 2, pp. 175-188.

Datta A., 2012, The Illegal City. Space, Law and Gender in a Delhi Squatter Settlement, Farnham (UK)/Burlington (USA), Ashgate.

DDA, 1957, Work studies relating to the preparation of the Master Plan for Delhi, Vol. 1, New Delhi, Delhi Development Authority.

DDA, 2007, Master Plan for Delhi 2021, Delhi, Delhi Development Authority [As Notified on 7th February, 2007, Vide S.O. No 141 published in: Gazette of India Extraordinary, Part II - Section 3 - Sub-Section (ii)].

Deshingkar P., Farrington J. (ed.), 2009, Circular Migration and Multilocational Livelihood Strategies in Rural India. Delhi, Oxford University Press.

Dewan Verma G., 2002, Slumming India. A chronicle of slums and their saviours, Delhi, Penguin.

Dupont V., 2004, "Socio-spatial differentiation and residential segregation in Delhi: a question of scale ?", Geoforum, 35, 2, pp. 157-175.

Dupont V., 2008, "Slum demolition in Delhi since the 1990s: An appraisal", Economic and Political Weekly, 43, 28, pp. 79-87.

Dupont V., 2009, «Enquêtes auprès de nouveaux nomades urbains: les victimes de la politique d'éradication des bidonvilles à Delhi (Inde) », Communication au XXVI ${ }^{\mathrm{e}}$ Congrès International de la Population, Marrakech, 27 septembre - 2 octobre 2009, UIESP, http://iussp2009.princeton.edu/papers/91337

Dupont V., 2010, «Création de nomades urbains et appauvrissement. Impact des politiques d'éradication des camps de squatters à Delhi », Revue Tiers Monde [Mobilité et pauvretés. Les villes interrogées], 201, pp. 25-45. 
Dupont V., 2011, "The dream of Delhi as a global city", International Journal of Urban and Regional Research, 35, 3, pp. 533-554.

Dupont V., Banda S., Vaidya Y., Gowda S., 2014, "Unpacking Participation in Kathputli Colony. Delhi's First Slum Redevelopment Project, Act I", Economic and Political Weekly, 49, 24, pp. 39-47.

Dupont V., Ramanathan U., 2007, «Du Traitement des slums à Delhi. Politiques de "nettoyage" et d'embellissement ", in Dupont V., Heuzé D. (eds), La ville en Asie du Sud: Analyse et mise en perspective, Paris, EHESS, coll. « Purushartha », 26, pp. 91-131.

Dupont V., Ramanathan U., 2008, "The Courts and the Squatter Settlements in Delhi. Or the Intervention of the Judiciary in Urban 'Governance' ", in Baud I., de Wit J. (eds), New Forms of Urban Governance in India. Shifts, Models, Networks, and Contestations, New Delhi, Sage, pp. 312-343.

Felstiner W., Abel R., Sarat A., 1980-81, "The emergence and transformation of disputes: Naming, blaming, claiming...", Law \& Society Review, 15, 3/4, pp. 631-654.

Ghertner A., 2010, "Calculating without numbers: aesthetic governmentality in Delhi's slums", Economy and Society, 39, 2, pp. 185-217.

Ghertner A., 2015, Rule by Aesthetics. World-class City Making in Delhi, Delhi, Oxford University Press.

Gilbert A., 2007, “The return of the slum: does language matter?”, International Journal of Urban and Regional Research, 31, 4, pp. 697-713.

GNCTD, 2003-04, "Relocation of JJ Squatters", Slum and Jhuggi-Jhopri Department (Municipal Corporation of Delhi), in National Capital Territory of Delhi. Annual Plan 20032004, vol. II [Scheme wise plan outlay], Delhi, Planning Department, Government of National Capital Territory of Delhi, pp. 1353-1367.

Gusfield J., 1986 [1963], Symbolic crusade. Status politics and the American temperance movement, Champaign, University of Illinois Press.

Harvey D., 2008, “The right to the city”, New Left Review, 53, September-October, pp. 23-40.

High Court of Delhi, 2002, “Okhla Factory Owners' Association vs Government of NCT of Delhi", Civil Writ Petition No. 4441/94 and 2112/2002 decided by the High Court of Delhi on 29 November 2002.

High Court of Delhi, 2010, "Sudama Singh \& others vs Government of Delhi", Civil Writ Petition No. 8904/2009, 7735/2007, 7317/2009 and 9246/2009, Judgement pronounced on 11 February 2010.

HLRN, 2009, Acts of Commission, Acts of Omission. Housing and land rights and the Indian State, New Delhi, Housing and Land Rights Network-Habitat International Coalition. 
HLRN, 2011, Planned Dispossession: Forced Evictions and the 2010 Commonwealth Games, Fact Finding Report Mission 14, New Delhi, Housing and Land Rights Network - Habitat International Coalition.

HLRN, 2014, Forced to the fringes. Disasters of 'Resettlement' in India, New Delhi, Housing and Land Rights Network - Habitat International Coalition.

HLRN-PUCL, 2013, Governance by denial. Forced eviction and demolition of homes in Ejipura/Koramamgala, Bangalore, Final Report of a fact-finding mission, New Delhi/ Bangalore, Housing and Land Rights Network/People's Union for Civil Liberties.

IGSSS, 2012, The Unsung CityMakers. A Study of the Homeless Residents of Delhi, New Delhi, Indo Global Social Service Society.

IPTEHR, 2005, Bulldozing Rights - A Report on the Forced Evictions and Housing Policies for the Poor in Mumbai, Mumbai, Indian People's Tribunal on Environment and Human Rights.

Jagmohan, 1978, Island of Truth, Delhi, Vikas.

Kothari M., 2003, "The human rights to adequate housing - India's commitment and the struggle towards realization", Journal of The National Human Rights Commission, India, 2, pp. 133-147.

Menon-Sen K., Bhan G., 2008, Swept off the Map. Surviving Eviction and Resettlement in Delhi, Delhi, Yodapress.

Neveu E., 2015, Sociologie politique des problèmes publics, Paris, A. Colin.

Nijman J., 2008, “Against the odds: Slum rehabilitation in neoliberal Mumbai”, Cities, 25, 2, pp. 73-85.

ONU-Habitat, Nations Unies-Haut Commissariat Droits de l'Homme (2014), Les expulsions forcées, Fiche d'information No 25/ Rev. 1, New York/Genève, Nations Unies.

Raman B., 2015, "The politics of property in land: new planning instruments, law and popular groups in Delhi", Journal of South Asian Development, 10, 3, pp. 369-395.

Roy A., 2005, "Urban informality: toward an epistemology of planning", Journal of the American Planning Association, 71, 2, pp. 147-158.

Roy A., 2011, "Slumdog cities : rethinking subaltern urbanism", International Journal for Urban and Regional Research, 35, 2, pp. 223-238.

Sassen S., 2013, Expulsions. When Complexity Produces Elementary Brutalities. Cambridge, M.A., Harvard University Press.

SCC, 2000, « Almitra Patel vs Union of India », Supreme Court Cases, vol. 2, pp. 679-690. 
Shah Commission of Enquiry, 1978, Interim Report II, Delhi, Government of India. https://archive.org/details/ShahCommissionOfInquiryInterimReportII Site consulté en septembre 2017.

Srishti, 2002, Report on Recycling Responsibilities, Traditional Systems and New Challenges of Urban Waste management in India, New Delhi, Srishti.

Weinstein L., 2008, "Mumbai's development mafias: Globalization, organized crime and land development", International Journal of Urban and Regional Research, 32,1, pp. 23-39. 